\title{
Does pre-resection endoscopic third ventriculostomy prevent the need for post-resection CSF diversion after pediatric posterior fossa tumor excision? A historical cohort study and review of the literature
}

\author{
*Harishchandra Lalgudi Srinivasan, FRCS(Neuro Surg), ${ }^{1,3}$ Mitchell T. Foster, MBChB, ${ }^{2}$ \\ Kirsten van Baarsen, MD, PhD, ${ }^{1}$ Dawn Hennigan, MSc, ${ }^{1}$ Benedetta Pettorini, MD, ${ }^{1}$ and \\ Conor Mallucci, FRCS(SN) ${ }^{1}$
}

1Department of Neurosurgery, Alder Hey NHS Foundation Trust, Liverpool; '2Department of Neurosurgery, Walton Centre NHS Foundation Trust, Liverpool, United Kingdom; and ${ }^{3}$ Department of Paediatric Neurosurgery, Tel Aviv Sourasky Medical Center, Tel Aviv, Israel

\begin{abstract}
OBJECTIVE Children with posterior fossa tumors (PFTs) may present with hydrocephalus. Persistent (or new) hydrocephalus is common after PFT resection. Endoscopic third ventriculostomy (ETV) is sometimes performed prior to resection to 1) temporize hydrocephalus prior to resection and 2) prophylactically treat post-resection hydrocephalus. The objective of this study was to establish, in a historical cohort study of pediatric patients who underwent primary craniotomy for PFT resection, whether or not pre-resection ETV prevents the need for post-resection CSF diversion to manage hydrocephalus.
\end{abstract}

METHODS The authors interrogated their prospectively maintained surgical neuro-oncology database to find all primary PFT resections from a single tertiary pediatric neurosurgery unit. These data were reviewed and supplemented with data from case notes and radiological review. The modified Canadian Preoperative Prediction Rule for Hydrocephalus (mCPPRH) score was retrospectively calculated for all patients. The primary outcome was the need for any form of postoperative CSF diversion within 6 months of PFT resection (including ventriculoperitoneal shunting, ETV, external ventricular drainage [EVD], and lumbar drainage [LD]). This was considered an ETV failure in the ETV group. The secondary outcomes were time to CSF diversion, shunt dependence at 6 months, and complications of ETV. Statistical analysis was done in RStudio, with significance defined as $p<0.05$

RESULTS A total of 95 patients were included in the study. There were 28 patients in the ETV group and 67 in the nonETV group. Patients in the ETV group were younger (median age 5 vs 7 years, $p=0.04$ ) and had more severe preoperative hydrocephalus (mean frontal-occipital horn ratio 0.45 vs 0.41 in the non-ETV group, $p=0.003$ ) and higher $\mathrm{mCPPRH}$ scores (mean 4.42 vs $2.66, p<0.001$ ). The groups were similar in terms of sex and tumor histology. The overall rate of post-resection CSF diversion of any kind (shunt, repeat ETV, LD, or EVD) in the entire cohort was $25.26 \%$. Post-resection CSF diversion was needed in $32 \%$ of patients in the ETV group and in $22 \%$ of the patients in the non-ETV group (p $>0.05)$. Shunt dependence at 6 months was seen in $21 \%$ of the ETV group and $16 \%$ of the non-ETV group $(p>0.05)$. The median time to ETV failure was 9 days. ETV failure correlated with patients with ependymoma $(p=0.02)$. Children who had ETV failure had higher mCPPRH scores than the ETV success group (5.67 vs 3.84, $p=0.04$ ).

CONCLUSIONS Pre-resection ETV did not reliably prevent the need for post-resection CSF diversion. ETV was more likely to fail in children with ependymoma and those with higher mCPPRH scores. Based on the findings of this study, the authors will change the practice at their institution; pre-resection ETV will now be performed based on a newly defined protocol.

https://thejns.org/doi/abs/10.3171/2019.12.PEDS19539

KEYWORDS persistent hydrocephalus; posterior fossa tumor; endoscopic third ventriculostomy; ETV; oncology ABBREVIATIONS CPA = cerebellopontine angle; ETV = endoscopic third ventriculostomy; EVD = external ventricular drainage; FOHR = frontal-occipital horn ratio; ioMRI =
intraoperative MRI; mCPPRH = modified Canadian Preoperative Prediction Rule for Hydrocephalus; PFT = posterior fossa tumor; VP = ventriculoperitoneal.

SUBMITTED September 15, 2019. ACCEPTED December 16, 2019.

INCLUDE WHEN CITING Published online February 21, 2020; DOI: 10.3171/2019.12.PEDS19539.

${ }^{*}$ H.L.S. and M.T.F. contributed equally to this study. 
$\mathrm{T}$ HE association between hydrocephalus and posterior fossa tumors (PFTs) in children is well established. There is a high incidence of moderate to severe hydrocephalus associated with pediatric PFTs at presentation (60\%-90\%), 5,7,12,18,20,24,27 which is thought to be secondary to the tumor obstructing outflow from the fourth ventricle. However, hydrocephalus persists in $16 \%-$ $33 \%$ of children even after the tumor is resected. $5,7,8,10,12,17,18$, 20,23,24 Why this hydrocephalus persists (or newly develops) after removal of the obstructive tumor is unclear, but various reasons have been implicated..$^{8,13,16,24}$ Initially, there is believed to be an "adaptation period" in CSF flow for up to 2 weeks after PFT resection while CSF pressures recalibrate. ${ }^{3}$ Purported mechanisms include disruption of the subarachnoid spaces, increasing flow across the pacchionian granules, or progressive dilation of stenosed venous sinuses. ${ }^{3,6}$ Post-resection blood and debris can migrate and obstruct CSF pathways or preoperatively placed shunts or ventriculostomies in what is described as the "snow globe effect." 25 Later, disease progression (tumor recurrence or metastasis) and complications of adjuvant therapy may contribute to delayed hydrocephalus.

Various management strategies are used in children presenting with PFT-associated hydrocephalus; CSF diversion can be achieved with external ventricular drainage (EVD), ventriculoperitoneal (VP) shunting, or endoscopic third ventriculostomy (ETV). Alternatively, the surgeon can proceed directly to tumor resection to primarily reopen CSF pathways. ${ }^{24}$ Whether to perform CSF diversion (and which diversion to perform) prior to tumor resection or after resection, or to simply monitor for post-resection hydrocephalus, continues to be debated. ${ }^{24}$ Pre-resection techniques to divert CSF prior to resection have been advocated by some to reduce postoperative morbidity and mortality. ${ }^{1}$ Notably, since the early 2000 s, pre-resection ETV has been adopted by many pediatric neurosurgery centers (Alder Hey included) in an effort to reduce the burden of hydrocephalus perioperatively. ${ }^{2,4,11,23}$ In addition to treating the hydrocephalus at presentation, the major purported benefit of pre-resection ETV is to reduce the incidence of post-resection hydrocephalus (from $10 \%-40 \%$ to $6 \%-15 \%) .^{2,4,13,22,23}$ The disadvantages are that ETV is an invasive procedure with theoretical highrisk complications, including major hemorrhage, stroke, and brainstem injury., ${ }^{4}$ It is also considered to be more technically complex than the alternative of EVD. There are several other proposed advantages and disadvantages of performing an ETV prior to tumor excision, which are presented in Table 1.

The objective of this study was to establish, in a historical cohort study of pediatric patients undergoing primary craniotomy for PFT resection, whether pre-resection ETV reduces the need for post-resection CSF diversion to manage hydrocephalus.

\section{Methods \\ Data Collection}

This was a historical cohort study involving the retrospective review of a prospectively maintained departmental database, supplemented with case note review. The
TABLE 1. Advantages and disadvantages of pre-resection ETV

\begin{tabular}{|c|c|}
\hline $\begin{array}{c}\text { Advantages of ETV Prior to PFT } \\
\text { Resection }\end{array}$ & $\begin{array}{c}\text { Disadvantages of ETV Prior to } \\
\text { PFT Resection }\end{array}$ \\
\hline $\begin{array}{c}\text { Reduction in need for } \\
\text { post-resection shunt } 2,4,9,13,23\end{array}$ & $\begin{array}{l}\text { Inherent complications } \\
\text { associated w/ ETV } 4,9\end{array}$ \\
\hline $\begin{array}{l}\text { Alleviate hydrocephalus prior to } \\
\text { resection }\end{array}$ & $\begin{array}{l}\text { May be technically challenging } \\
\text { due to distorted anatomy } 4,9\end{array}$ \\
\hline $\begin{array}{l}\text { Reduce ICP \& make surgical } \\
\text { approach less challenging }\end{array}$ & $\begin{array}{l}\text { Risk of upward herniation/ } \\
\text { intratumoral bleed }\end{array}$ \\
\hline $\begin{array}{l}\text { Less post-resection CSF leak \& } \\
\text { pseudomeningocele } e^{9,23}\end{array}$ & $\begin{array}{l}\text { Inability to titrate ICP in compari- } \\
\text { son } w / E V D^{27}\end{array}$ \\
\hline $\begin{array}{l}\text { Reduction in pseudobulbar } \\
\text { palsy9,23 }\end{array}$ & $\begin{array}{l}\text { Risk of failure if ventriculostomy } \\
\text { becomes occluded by } \\
\text { post-resection blood or debris } \\
\text { (the "snow globe effect") }{ }^{25}\end{array}$ \\
\hline
\end{tabular}

$\mathrm{ICP}=$ intracranial pressure .

STROBE guidelines were used in the reporting of this study. ${ }^{28}$

Institutional review board (Alder Hey) approval was obtained. The database was interrogated to identify all pediatric patients (age $0-18$ years) who underwent craniotomy for the resection of a newly diagnosed PFT. The following patients were excluded: children with revision surgery, patients who received biopsy alone, any patient who received a pre-resection VP shunt, patients older than 18 years at the time of surgery, and any child receiving neoadjuvant chemotherapy (not planned for resection).

Patient demographics, surgical details, and postoperative complications between 0 and 30 days had been prospectively recorded. Details of perioperative CSF diversion surgery were retrospectively abstracted from surgical case notes. We chose variables a priori that were anticipated to correlate with post-resection hydrocephalus risk. These were tumor location (stratified as midline, paramedian, brainstem, or cerebellopontine angle [CPA]), tumor size (maximum diameter measured on MRI), the severity of preoperative hydrocephalus (measured with the frontal-occipital horn ratio [FOHR]), tumor nature (cystic or metastatic), tumor extension to the foramen magnum, presence of post-resection intraventricular blood on MRI, extent of tumor resection, and presence of postoperative CSF leak. We also quantified each child's modified Canadian Preoperative Prediction Rule for Hydrocephalus (mCPPRH) score. ${ }^{14,20}$

\section{Institutional Surgical Management of PFTs and Associated Perioperative Hydrocephalus}

This study comprises patients from a single tertiary pediatric neurosurgery center (Alder Hey NHS Foundation Trust). Children presenting with PFTs are generally referred from peripheral centers and undergo a full tumor protocol MRI of the brain and spine soon after arrival to our center. All of these children are discussed in the neuro-oncology multidisciplinary team at the regional or national level, depending on the provisional diagnosis prior to tumor resection, considering the benefit of a clear predefined surgical strategy. From the point of referral, a 
child is taken for tumor resection usually within 1 week. All children are started on dexamethasone to reduce vasogenic edema. Tumor resection is performed under general anesthesia, with patients prone (or occasionally lateral for CPA tumors) and with intraoperative MRI (ioMRI) to confirm the extent of resection. Surgery is not performed in the sitting position. PFT resection is almost universally performed by the lead neuro-oncology surgeon (C.M.).

Sometimes children with PFTs present with acutely symptomatic hydrocephalus, and CSF diversion cannot wait. One approach to manage this is to place an external ventricular drain at this point. In some circumstances, this has to be done as an emergency, out of hours. However, in our unit, if the patient is stable enough, we prefer to perform an ETV to safely manage the hydrocephalus and allow preparation for surgery with ioMRI. There are 2 main reasons for the preference for ETV over EVD. First, ETV is thought to have a lower risk of CSF infection. Second, ETV is thought to have a secondary benefit of reducing post-resection hydrocephalus (the hypothesis that forms the basis of this paper). There were no predefined protocols as to when ETV should be performed; it was based on the duty consultant neurosurgeon's clinical digression, balancing the patient's clinical condition, whether ETV was anatomically feasible, and the time to the next ioMRI capable list. Unfortunately, it is not possible to retrospectively ascertain the nuances of this decision-making process.

ETV is performed under general anesthesia with the patient positioned supine. A frontal burr hole (usually right sided) is created, the dura is opened, a peel-away catheter is inserted into the lateral ventricle, and a rigid endoscope is inserted. The floor of the third ventricle is identified, and a balloon dilator is used to fashion the ventriculostomy. If the ventriculostomy was considered intraoperatively to be at risk of immediate postoperative failure, a rescue EVD was placed in the lateral ventricle and kept closed by default after the ETV. This allowed for emergency external CSF drainage if required. If CSF had to be drained from the rescue EVD, it was considered as an ETV failure.

After tumor resection, children were later considered for CSF diversion if they experienced any of the following: clinical deterioration from hydrocephalus, persistent CSF leak that did not respond to simple wound reinforcement with sutures, or a tense pseudomeningocele with moderate to severe hydrocephalus. In children with a CSF leak or pseudomeningocele without ventriculomegaly, local wound reinforcement stitches or lumbar puncture was performed.

\section{Statistical Analysis}

Preoperative ETV status was categorized as one of the 3 following separate types: 1) not performed, 2) performed with a successful ventriculostomy fashioned, or 3) attempted but for whatever reason third ventriculostomy was unsuccessful intraoperatively. This was then dichotomized for analysis as ETV successfully performed or ETV not performed (which included the unsuccessful attempts). The rationale for this was to understand the role of the ventriculostomy itself, rather than the intention to perform an ETV. For completeness, an analysis of the intention to perform an ETV was also examined.
The primary outcome of this study was the need for CSF diversion within 6 months of tumor resection (including ETV, EVD, lumbar drainage, or shunt of any kind). If the patient had an ETV performed preoperatively and met the primary outcome criteria, they were considered to have ETV failure. Lumbar puncture was not considered to be a sufficiently invasive procedure to qualify for this outcome measure. Furthermore, many children receive a postoperative lumbar puncture as part of their diagnostic workup, and it is not always possible to retrospectively establish if a lumbar puncture was for therapeutic, diagnostic, or both purposes.

The secondary outcomes were time to first post-resection CSF diversion, the need for shunting of any kind within 6 months of resection, and direct complications of ETV.

Data analysis was performed using RStudio (RStudio Team, 2015). Univariate analysis was performed to assess prognostic markers for the primary outcome. In continuous, normally distributed variables, the Student t-test was used; the chi-square test was used for categorical variables; and the Wilcoxon signed-rank test was used for nonparametric ordinal data. A Cox proportional hazards model was used to compare time to CSF diversion between the two groups. This was adjusted for preoperative hydrocephalus severity (as measured with the FOHR) and tumor size (as measured by maximum diameter). Significance was defined as $\mathrm{p}<0.05$.

\section{Results}

Between January 2008 and December 2018, there were 170 PFT-related surgeries. Of these, 95 were eligible for inclusion into the study based on the exclusion criteria. A summary of patient inclusion, treatment pathways, and outcomes is presented in Fig. 1.

\section{Group Baseline Comparison}

The groups were heterogeneous regarding group size, age, severity of preoperative hydrocephalus, tumor size, and mCPPRH score (Table 2). There was no significant difference in sex or histology between the two groups. These results indicate that (as would be anticipated based on our unit's clinical practice) patients with larger tumors, more significant hydrocephalus at presentation (as measured by FOHR), and higher risk of post-resection hydrocephalus (as measured by the mCPPRH score) were more likely to receive pre-resection ETV (Table 2). There were no other significant differences between the two groups (we also compared tonsillar descent, extension to the foramen magnum, metastasis, and solid vs cystic tumors).

\section{Surgical Treatment Pathways}

Figure 1 shows a graph of patient inclusion, their treatment pathways (pre- and postoperative CSF diversion), and CSF dependence outcomes.

\section{Preoperative ETV Group}

A total of 31 ETVs were attempted; 28 were successful technically $(90.3 \%)$ and 3 were unsuccessful intraopera- 


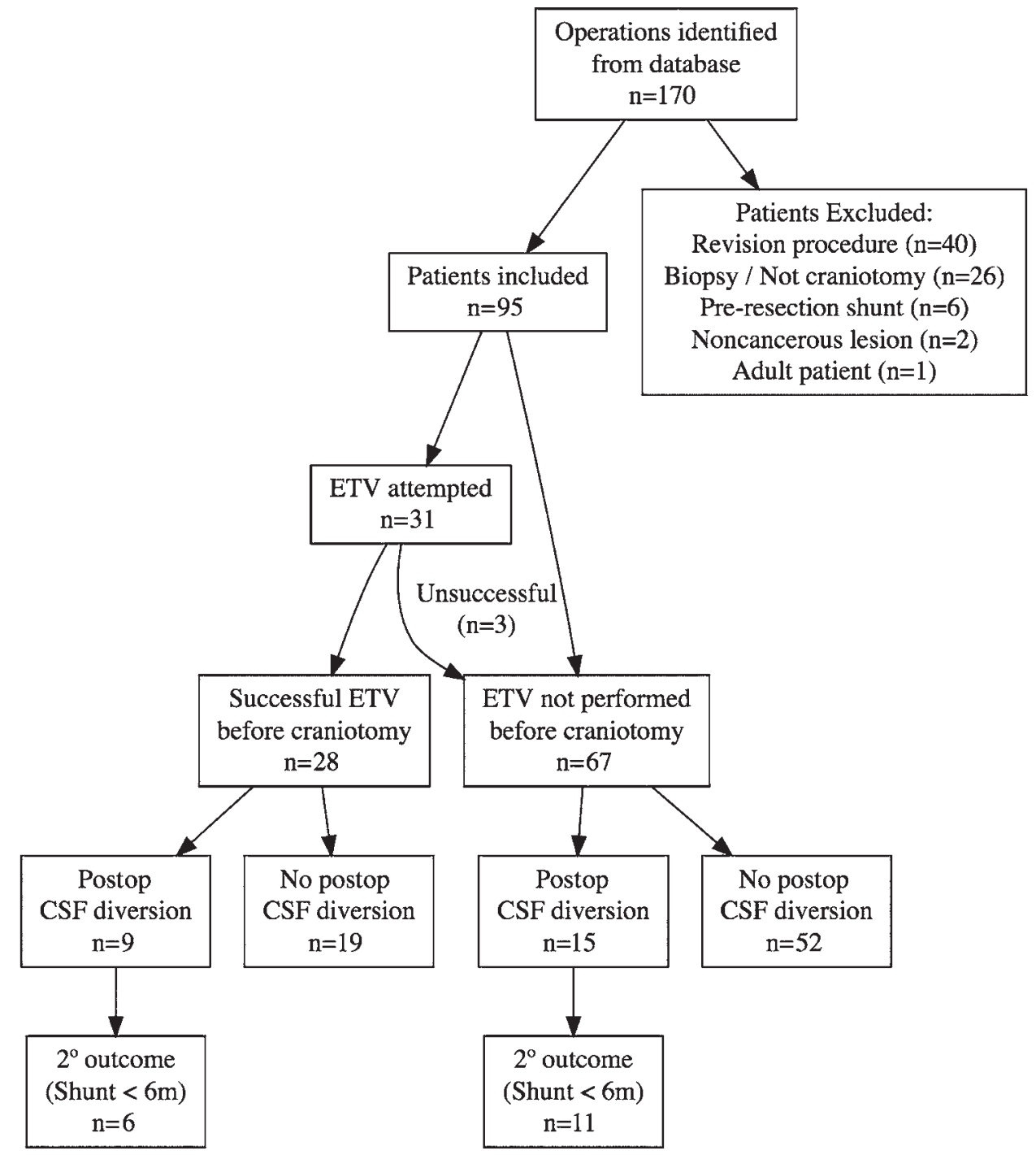

FIG. 1. Flow diagram of patient screening and exclusion, and subsequent treatment pathways in the included patients. The cohort was divided into ETV and non-ETV groups, and outcomes were measured. The primary outcome was any CSF diversion within 6 months $(<6 \mathrm{~m})$ of resection. The secondary $\left(2^{\circ}\right)$ outcome was insertion of a shunt within 6 months of resection.

tively $(9.7 \%)$. The reasons for failure to establish a ventriculostomy were 1) distorted anatomy of the third ventricle, 2) poor visualization due to turbid CSF, and 3) intraoperative bleeding. These patients were therefore considered not to have had an ETV, despite the surgical procedure with intent to perform one. A post-ETV rescue external ventricular drain was placed in 2 children because CSF flow across the newly fashioned ventriculostomy was felt to be inadequate. In both cases, this external ventricular drain did not need to be opened and was removed within 72 hours, and no further CSF diversion was necessary. The median time between ETV and resection was 3 days (IQR 3 days, range $0-14$ days).

\section{Primary Outcome}

Table 3 shows the primary outcome of this study (the requirement of surgical CSF diversion within 6 months of PFT resection) for the ETV group versus the non-ETV group. There was a trend toward more post-resection CSF diversion in the ETV group (i.e., ETV failure) compared with the non-ETV group, although this was not statistically significant. The rate of any post-resection CSF diversion (shunt, repeat ETV, lumbar drainage, or EVD) in the entire cohort was $25.26 \%$. The reasons for ETV failure included blood over the ventriculostomy $(\mathrm{n}=2)$, stoma closure (n $=1)$, meningitis requiring $\operatorname{EVD}(\mathrm{n}=1)$, and reason not known $(n=5)$.

\section{Secondary Outcomes}

Shunt Dependence at 6 Months

The rate of post-resection shunt dependence at 6 months was $17.89 \%$ for the entire cohort. It was $16 \%$ in the non-ETV group and $21 \%$ in the ETV group (Table 3). This difference was not statistically significant. 
TABLE 2. Patients and tumor characteristics

\begin{tabular}{|c|c|c|c|c|}
\hline & $\begin{array}{c}\text { All } \\
\text { Patients }\end{array}$ & $\begin{array}{l}\text { ETV } \\
\text { Group }\end{array}$ & $\begin{array}{l}\text { Non-ETV } \\
\text { Group }\end{array}$ & p Value \\
\hline No. of patients & 95 & 28 & 67 & \\
\hline Sex & & & & $0.61^{*}$ \\
\hline F/M ratio & $0.79: 1$ & $1.15: 1$ & $0.68: 1$ & \\
\hline $\begin{array}{l}\text { No. of male } \\
\text { patients }\end{array}$ & 53 & 13 & 40 & \\
\hline Age, yrs & & & & $0.04 \uparrow$ \\
\hline Median (IQR) & $7(7)$ & $5(5)$ & $7(7)$ & \\
\hline Mean (SD) & $7.15(4.56)$ & $5.68(4.23)$ & $7.77(4.58)$ & \\
\hline $\begin{array}{l}\text { Mean preop FOHR } \\
\text { (SD) }\end{array}$ & $0.42(0.08)$ & $0.45(0.05)$ & $0.41(0.08)$ & $0.003 \uparrow$ \\
\hline $\begin{array}{l}\text { Mean maximum } \\
\text { tumor diameter (SD) }\end{array}$ & $4.73(1.52)$ & $5.39(1.14)$ & $4.46(1.58)$ & $0.002 \dagger$ \\
\hline mCPPRH score ${ }^{14}$ & & & & $<0.001 \ddagger$ \\
\hline Median (IQR) & $3(3)$ & $4(3)$ & $3(3)$ & \\
\hline Mean (SD) & $3.18(2.15)$ & $4.43(1.95)$ & $2.66(2.02)$ & \\
\hline \multicolumn{5}{|l|}{ Histology } \\
\hline Medulloblastoma & 31 & 8 & 23 & \multirow{3}{*}{$0.47^{*}$} \\
\hline Ependymoma & 20 & 7 & 13 & \\
\hline $\begin{array}{l}\text { Pilocytic astrocy- } \\
\text { toma }\end{array}$ & 30 & 9 & 21 & \\
\hline Other & 14 & 4 & 10 & \\
\hline \multicolumn{5}{|l|}{ Predominant location } \\
\hline Midline & 48 & 14 & 34 & \multirow{4}{*}{$0.67^{*}$} \\
\hline Brainstem & 4 & 2 & 2 & \\
\hline CPA & 11 & 2 & 9 & \\
\hline Paramedian & 32 & 10 & 22 & \\
\hline
\end{tabular}

\section{Time to Post-Resection CSF Diversion}

The median time to ETV failure was 9 days (IQR 8 days, range $0-14$ days). Figure 2 graphs a Cox proportional hazards model of post-resection CSF diversion up to 6 months (182 days), adjusted for pre-resection mCPPRH score. This identified no statistically significant difference between the two groups.

\section{Complications of ETV}

The complications with performing an ETV prior to tumor resection can be considered in 3 phases: intraoperative, before tumor resection, and after tumor resection. There were 3 intraoperative cases of procedural failure, as discussed above. There were no other intraoperative complications.

The conditions of 2 patients deteriorated after their ETV but prior to tumor resection, meaning their tumor resection had to be performed earlier than planned. In one child there was radiological evidence of post-ETV hemorrhage within the tumor, but it is not clear if this was due to the ETV or natural progression of the tumor.

The rate of post-resection CSF leak was not significant-
TABLE 3. Primary and secondary outcomes in the ETV and non-ETV groups

\begin{tabular}{lccc}
\hline \multirow{1}{*}{ Outcome } & \multicolumn{2}{c}{ No. of Patients (\%) } & $p$ \\
\cline { 2 - 3 } & No-ETV & ETV & Value \\
\hline $\begin{array}{l}\text { Primary outcome: any CSF diversion } \\
\text { at 6 mos }\end{array}$ & $15(22.39)$ & $9(32.14)$ & 0.46 \\
\hline $\begin{array}{l}\text { Secondary outcome: shunt at 6 mos } \\
\text { Seconn }\end{array}$ & $11(16.42)$ & $6(21.43)$ & 0.77 \\
\hline
\end{tabular}

ly different between the two groups; $10.71 \%$ in the ETV group and $13.43 \%$ in the non-ETV group $(\mathrm{p}=0.98)$.

The overall rate of infection was $1 \%$ for the entire cohort. The rate of infection in the ETV group $(1 / 31,3 \%)$ was not statistically different from that in the non-ETV group (0\%). The one case of infection presented as meningitis at 2 weeks after resection. This patient required an EVD for intrathecal antibiotics but did not need the EVD for the purpose of CSF diversion. It is not clear whether the ETV or the resection was causative.

One patient in the ETV group developed a subdural hygroma needing a subduroperitoneal shunt after tumor resection $(\mathrm{n}=1)$.

\section{Can We Identify Factors That Are Prognostic for ETV Failure?}

We evaluated whether ETV success could be predicted. Table 4 shows a comparison of variables chosen a priori and their association with ETV failure or success in our cohort. Of these variables, the only independent factor that significantly correlated with ETV success was histology: ETV failed in $71 \%$ of patients with ependymoma (Table 4). No patients with a pilocytic astrocytoma had ETV failure.

Table 4 also shows that the group with ETV failure had significantly higher mCPPRH scores at presentation. Therefore, although this was not the score's original purpose, we evaluated whether the score could be repurposed to predict the success of pre-resection ETV in preventing post-PFT resection hydrocephalus. In line with previous publications, the mCPPRH score was used to dichotomize patients into high-risk (mCPPRH score 5-10) or low-risk (mCPPRH score 0-4) groups for post-resection hydrocephalus. When applied to our ETV cohort, the dichotomized mCPPRH score had a specificity of $44.44 \%$, sensitivity of $78.95 \%$, positive predictive value of $75 \%$, and negative predictive value of $50 \%$ with respect to predicting ETV failure.

Within the ETV group, there were 8 patients who would have been considered at high risk of post-resection hydrocephalus on the mCPPRH scale. Of these, 3 patients needed a shunt, all of whom had intraventricular blood on their post-resection MRI scan. Because of the small numbers, this association was not statistically significant. However, our interpretation is that patients who are in the high-risk group and are treated with an ETV are vulnerable to ventriculostomy closure if there is post-resection intraventricular hemorrhage via the snow globe effect. ${ }^{25}$

\section{Discussion}

We can consider ETV as having two main goals: 1) to 


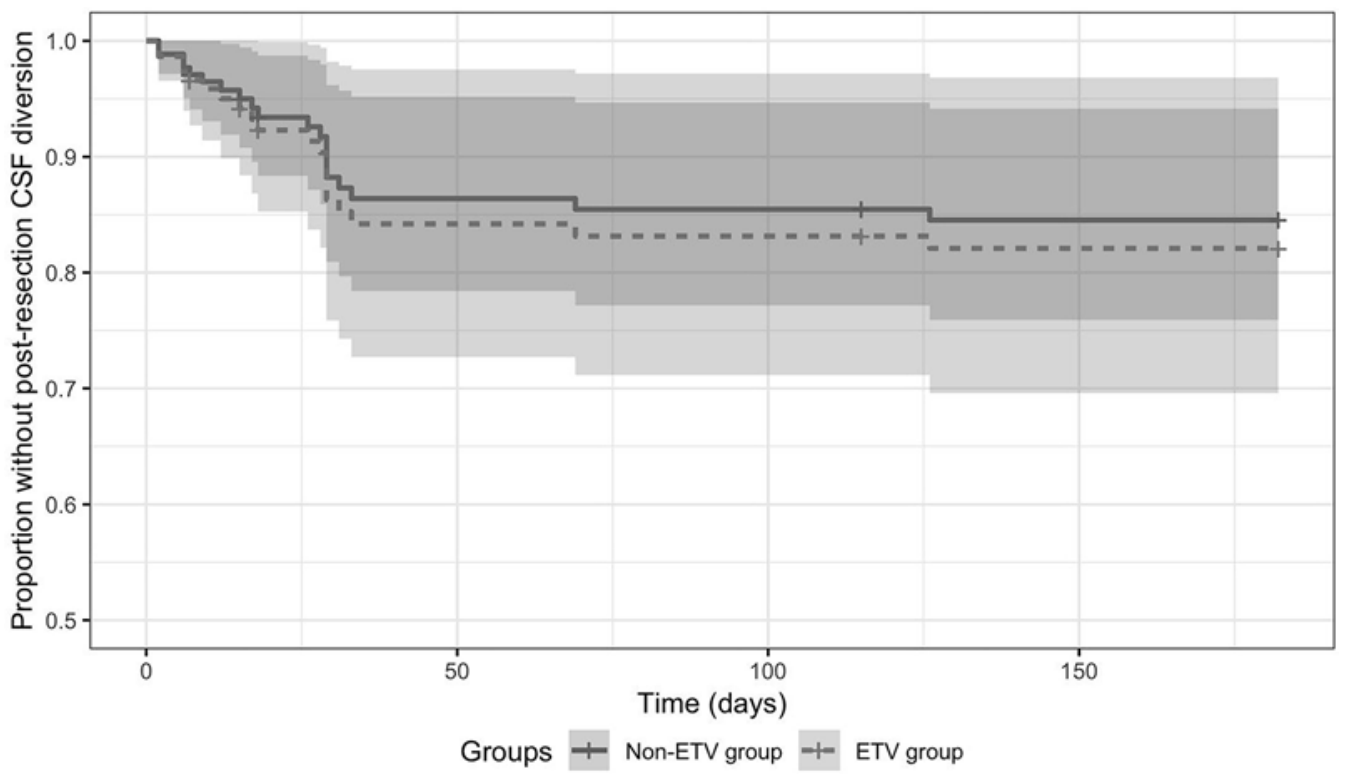

FIG. 2. Cox regression plot of primary outcome (post-resection CSF diversion at 6 months) in the pre-resection ETV versus nonETV groups. This was adjusted for preoperative hydrocephalus severity (as measured with FOHR) and tumor size (as measured by maximum diameter).

acutely treat pre-resection (presentation) hydrocephalus and 2) to prophylactically manage post-resection hydrocephalus. The primary objective of this study was to examine the latter goal, but our results for both goals merit discussion.

\section{ETV to Acutely Treat Pre-Resection (Presentation) Hydrocephalus}

ETV was generally effective in the management of pre-resection hydrocephalus. It was a reliable temporizing measure, providing time for complete workup and planning an effective surgical strategy. Our intraoperative ETV technical failure rate $(9.7 \%, \mathrm{n}=3 / 31)$ is higher than that reported in the literature $(3 \%-5 \%)$, but the reasons we encountered were comparable., , $, 922,23,29$ It is thought that even if ventriculostomy is technically feasible, the absence of pulsation across the ventriculostomy floor may be prognostic for ETV failure. ${ }^{21}$ In our series, 2 children with symptomatic hydrocephalus at presentation did not improve after ETV and required tumor resection earlier than planned. We can therefore consider these 5 patients who underwent surgery with the intent of ETV (5/31 attempted ETVs; $16 \%$ ) as a group in which ETV surgery failed to achieve this goal of managing presentation hydrocephalus.

Many units would insert an EVD at the time of or just before performing craniotomy to treat the associated hydrocephalus. This of course also requires surgical access to the ventricle (ventriculostomy) and will manage the immediate hydrocephalus preoperatively. It is a technically less challenging procedure, and one in which a procedural failure is less likely. One of the reasons cited for not performing EVD is to avoid potential associated infection with prolonged EVD (6\%-15\%), although this rate has decreased with the use of antibiotic-impregnated cath- eters. ${ }^{18,26}$ In our series, the overall infection rate for PFT resection was only $1 \%$, which may justify an avoidance of EVDs pre- and perioperatively.

\section{ETV to Prophylactically Manage Post-Resection Hydrocephalus}

ETV was not a reliable prophylactic measure in managing post-resection hydrocephalus. There was no statistically significant difference between the ETV group and the non-ETV group in terms of the primary outcome (any CSF diversion at 6 months) or the secondary outcomes (time to CSF diversion and shunt dependence at 6 months), although there was a non-statistically significant trend toward the ETV group needing more CSF diversion procedures. This may be because the ETV group had larger tumors with more severe hydrocephalus at presentation and a higher risk of post-resection hydrocephalus per the $\mathrm{mCPPRH}$ scale. The overall shunt rate for the entire cohort was $18 \%$.

The results of this study do not justify the routine use of ETV as a prophylactic measure to prevent post-resection hydrocephalus that is advocated by other authors. ${ }^{5,15,19} \mathrm{In}$ particular, our data indicate that the children who stand to gain the most from pre-resection ETV (i.e., children who are at high risk of post-resection hydrocephalus based on the mCPPRH score) do not actually see the intended benefit; ETV failure in the high-risk group was $37.5 \%(n=3 / 8)$. This interpretation should be taken with caution as this is only a single series, the sample size is small, there was no direct comparison with alternative surgical options (namely, EVD), and we applied the mCPPRH retrospectively.

There is also no evidence available in the literature to suggest which patients are likely to benefit from ETV if it is to be performed only in select patients. Because of the 
TABLE 4. Univariate analysis of factors that were considered a priori to potentially predict ETV failure

\begin{tabular}{|c|c|c|c|}
\hline Variable & $\begin{array}{c}\text { ETV } \\
\text { Success }\end{array}$ & $\begin{array}{l}\text { ETV } \\
\text { Failure }\end{array}$ & $\begin{array}{c}p \\
\text { Value }\end{array}$ \\
\hline \multicolumn{4}{|l|}{ Cystic nature of tumor } \\
\hline Cystic & $7(77.78)$ & $2(22.22)$ & \multirow{3}{*}{$0.678^{*}$} \\
\hline Solid & $11(64.71)$ & $6(35.29)$ & \\
\hline Mixed & $1(50)$ & $1(50)$ & \\
\hline Nonmetastatic lesion & $18(69.23)$ & $8(30.77)$ & \multirow{2}{*}{$>0.99^{*}$} \\
\hline Metastatic lesion & $1(50)$ & $1(50)$ & \\
\hline \multicolumn{4}{|l|}{ Histology } \\
\hline Ependymoma & $2(28.57)$ & $5(71.43)$ & \multirow{4}{*}{$0.006^{*}$} \\
\hline Medulloblastoma & $4(50)$ & $4(50)$ & \\
\hline Other & $4(100)$ & $0(0)$ & \\
\hline Pilocytic astrocytoma & $9(100)$ & $0(0)$ & \\
\hline \multicolumn{4}{|l|}{ Predominant location } \\
\hline Brainstem & $2(100)$ & $0(0)$ & \multirow{4}{*}{$0.137^{*}$} \\
\hline CPA & $1(50)$ & $1(50)$ & \\
\hline Midline & $7(50)$ & $7(50)$ & \\
\hline Paramedian & $9(90)$ & $1(10)$ & \\
\hline
\end{tabular}

\section{Tumor extension to foramen}

magnum

\begin{tabular}{|c|c|c|c|}
\hline No & $13(76.47)$ & $4(23.53)$ & \multirow{2}{*}{$0.424^{*}$} \\
\hline Yes & $6(54.55)$ & $5(45.45)$ & \\
\hline \multicolumn{4}{|l|}{ Hemorrhage } \\
\hline No & $18(69.23)$ & $8(30.77)$ & \multirow{2}{*}{$>0.99^{*}$} \\
\hline Yes & $1(50)$ & $1(50)$ & \\
\hline \multicolumn{4}{|l|}{ Postop intraventricular clot } \\
\hline No & $16(80)$ & $4(20)$ & \multirow{2}{*}{$0.084^{*}$} \\
\hline Yes & $3(37.50)$ & $5(62.50)$ & \\
\hline \multicolumn{4}{|l|}{ Extent of resection } \\
\hline Complete & $17(70.83)$ & $7(29.17)$ & \multirow{2}{*}{$0.804^{*}$} \\
\hline Subtotal & $2(50)$ & $2(50)$ & \\
\hline \multicolumn{4}{|l|}{ Post-resection CSF leak } \\
\hline No & $18(72)$ & $7(28)$ & \multirow{2}{*}{$0.483^{*}$} \\
\hline Yes & $1(33.33)$ & $2(66.67)$ & \\
\hline \multicolumn{3}{|l|}{ mCPPRH score } & \multirow{3}{*}{$0.4^{*}$} \\
\hline 5-10 (high risk) & $4(50)$ & $4(50)$ & \\
\hline 0-4 (low risk) & $15(75)$ & $5(25)$ & \\
\hline Mean mCPPRH score & 3.84 & 5.67 & $0.04 \dagger$ \\
\hline Mean age, yrs & 5.68 & 5.67 & $>0.99 \dagger$ \\
\hline Mean preop FOHR & 0.44 & 0.47 & $0.118 \dagger$ \\
\hline Mean maximum tumor diameter, $\mathrm{cm}$ & 5.26 & 5.67 & $0.384 \dagger$ \\
\hline
\end{tabular}

Values represent the number of patients (\%) unless stated otherwise. Boldface roman type indicates statistical significance at $p<0.05$. Boldface italic type indicates statistical significance at $p<0.01$.

${ }^{*}$ Chi-square test.

†t-test.

small sample size in this study, it was not possible to generate an accurate new prognostic model for pre-resection ETV success. However, ETV failure was found to be associated with ependymoma in our series, similar to the find- ings of El Beltagy et al. ${ }^{11}$ One hypothesis for this is that ependymoma and medulloblastoma may be associated with a prolonged adaptation period following their excision because of secondary inflammation of subarachnoid spaces. ${ }^{3,9}$ None of our other individual prognostic factors selected a priori correlated with ETV failure risk. We did identify that a higher mCPPRH score showed some correlation with ETV failure. The implication of this is that children with a high mCPPRH score are likely to need post-resection CSF diversion regardless of whether preresection ETV was performed, and therefore performing a pre-resection ETV may be an unjustifiable risk when compared with the alternative method of EVD.

\section{Study Limitations}

This study is limited because it is retrospective, the numbers are small, and the treatment cohorts are heterogeneous in multiple ways. Furthermore, the decision to offer ETV was not prospectively protocolized; it was dependent on one of 5 on-call pediatric neurosurgeons and their clinical decisions at the time. It was not possible to retrospectively define why ETV was considered because the decision-making process is nuanced. It is clear from our results that patients with more significant hydrocephalus were more likely to receive ETV, but this score (mCPPRH) was not necessarily applied at the time of clinical decision-making.

\section{Generalizability}

A systematic review on the role of perioperative ETV was published in 2017. ${ }^{8}$ We reviewed this publication and any publications since and identified 6 eligible papers for pooled analysis of pre-resection ETV in pediatric PFT (Table 5). 2,4,11,13,22,23 The papers were comparable in terms of patient age, tumor histology, location, metastasis, and extent of resection. However, they were heterogeneous in terms of the duration of follow-up, the definition of ETV failure, and the patient inclusion and exclusion criteria. For example, 3 papers $(50 \%)$ also included tumor biopsy alone after ETV (rather than resection), which we elected to exclude in our series..$^{2,22,23}$ A comparison of ETV failure rates between these papers is presented in Fig. 3. The mean failure rate for all published series is $15.91 \%$ (range 6\%-38\%). To have a comparable outcome measure with these papers, we present our secondary outcome (post-resection shunt) on this graph.

In our series, the median duration to ETV failure was 9 days after tumor resection, which was quicker than the pooled average of published series (25 days). ${ }^{8}$ In the literature, if an ETV is to fail, more than $50 \%$ will fail within 30 days of tumor resection. . $^{211,13,22,23}$ Within our series, $100 \%$ failed within this time frame. It is expected that the reasons for ETV failure beyond this short time period are heterogeneous and not directly related to the primary surgery, and may, for example, be due to disease recurrence, new metastasis, or adjuvant therapy. ${ }^{5}$

The two series with the lowest failure rates (SainteRose et al.: 6\%; El-Ghandour: 6.2\%) delayed resection by at least 1 week after ETV (Sainte-Rose et al.: mean 40 days; El-Ghandour: range 7-14 days). ${ }^{13,23}$ In the remaining series with failure rates of more than $10 \%$ (ours in- 
TABLE 5. Summary of pre-resection ETV outcomes in the literature

\begin{tabular}{|c|c|c|c|c|c|c|}
\hline Authors \& Year & $\begin{array}{l}\text { No. of } \\
\text { ETVs }\end{array}$ & Definition of Failure & $\begin{array}{l}\text { Failure } \\
\text { Rate }\end{array}$ & $\begin{array}{l}\text { Interval Btwn ETV } \\
\text { \& Resection (days) }\end{array}$ & $\begin{array}{l}\text { Position for } \\
\text { Resection }\end{array}$ & $\begin{array}{l}\text { Includes } \\
\text { Biopsies }\end{array}$ \\
\hline Sainte-Rose et al., 2001 & 67 & Postop hydrocephalus & $6 \%$ & 40 & $82 \%$ sitting & Yes $(4 \%)$ \\
\hline Ruggiero et al., 2004 & 20 & Need for VP shunt & $15 \%$ & $<7$ & Prone & Yes $(5 \%)$ \\
\hline Bhatia et al., 2009 & 37 & Postop hydrocephalus & $13.5 \%$ & 4.7 & Not described & No \\
\hline El Beltagy et al., 2010 & 40 & VP shunt $<1 \mathrm{yr}$ & $37.5 \%$ & $<7$ & Prone & No \\
\hline El-Ghandour, 2011 & 32 & Postop hydrocephalus & $6.2 \%$ & $7-14$ & Not described & No \\
\hline Azab et al., 2013 & 17 & Need for VP shunt & $11.8 \%$ & $2-4$ & Not described & Yes $(6 \%)$ \\
\hline \multirow{2}{*}{ Current series } & \multirow{2}{*}{31} & Any CSF diversion 6 mos post-resection & $32.1 \%$ & \multirow{2}{*}{$<7$} & \multirow{2}{*}{ Prone } & \multirow{2}{*}{ No } \\
\hline & & VP shunt $<6$ mos & $21 \%$ & & & \\
\hline
\end{tabular}

cluded), tumor resection was performed within 1 week of ETV. ${ }^{2,4,11,22}$ The implication is that a substantial delay to resection after ETV increases the likelihood of persistent ETV success. We hypothesize that this time delay allows the ETV stoma to establish and for CSF dynamics to recalibrate to persistent flow through it, reducing the likelihood of closure. This concept is theoretical and unable to be validated with our data. The role of the time interval between tumor resection and ETV in ETV success warrants further evaluation.

\section{Conclusions}

Pre-resection ETV is an accepted and commonly practiced technique to control symptomatic preoperative hydrocephalus. It also acts as a temporizing measure in children who otherwise cannot wait for a delayed planned surgery on an appropriate operation list after workup for complete imaging and multidisciplinary team discussion.

In our institution, preoperative ETV has resulted in avoiding EVD as an alternative and achieving a very low infection rate (1\%). However, overall, our institutional experience with ETV as a means of avoiding post-craniotomy CSF diversion was poor. We had high rates of procedural ETV failure, and pre-resection ETV did not reliably result in fewer post-resection CSF diversion procedures. One of the reasons to analyze these data was to try to learn

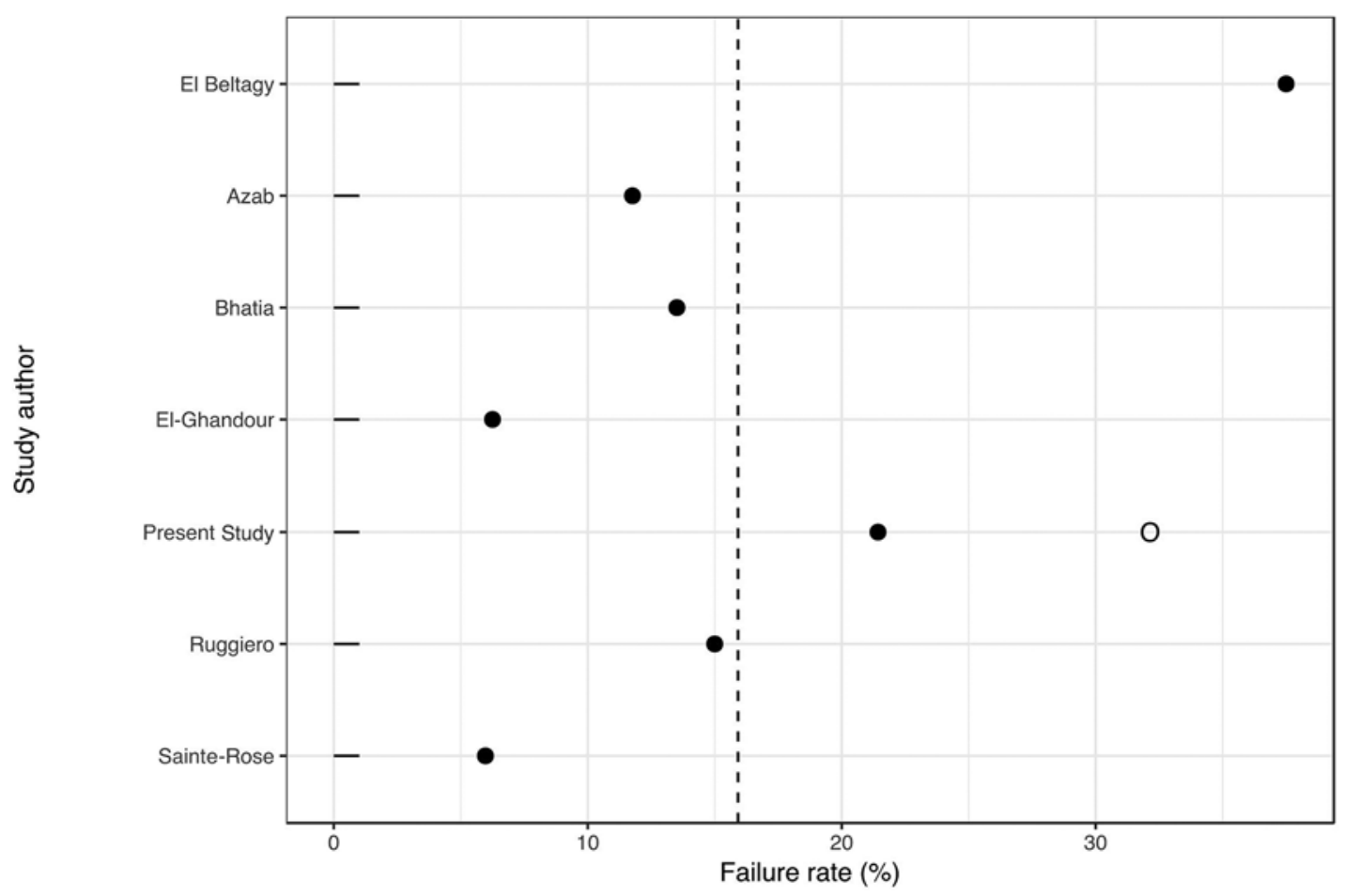

FIG. 3. Comparison of pre-resection ETV failure rates in published series. To have a comparable outcome measure with previously published series, we present both our secondary outcome (post-resection shunt within 6 months; black dots) and our primary outcome (any post-resection CSF diversion within 6 months; white dot) on this graph. The dotted line indicates the mean. 
from our experience and to introduce an evidence base and standardize practice within our department regarding CSF diversion both pre- and postoperatively.

The outcome of this work will change our practice regarding pre-resection hydrocephalus management. We recommend that ETV should be restricted to children with significant symptomatic hydrocephalus, and not just hydrocephalus presenting radiologically to avoid unnecessary ETV being performed. If the provisional diagnosis prior to resection is ependymoma, ETV will not be performed. Alternatives to preoperative ETV in PFT with symptomatic hydrocephalus include going straight to a more urgent craniotomy, with or without a perioperative external ventricular drain to cover the hydrocephalus and raised intracranial pressure. A pragmatic, patient-specific balance between early (even urgent) craniotomy and hydrocephalus management versus temporizing with preoperative CSF procedures to allow for the perfect workup and operating list needs to be struck. While in clinical practice revision posterior fossa craniotomy is common, the results of this study should only be considered valid to primary PFT resections.

Clearly, ETV has a role in managing pre-resection PFT hydrocephalus, but selecting appropriate patients is challenging. A larger, multicenter prospective study or pooled analysis of data from appropriate published series may be helpful in deriving an evidence-based decision tool.

\section{References}

1. Albright L, Reigel DH: Management of hydrocephalus secondary to posterior fossa tumors. J Neurosurg 46:52-55, 1977

2. Azab W, Al-Sheikh T, Yahia A: Preoperative endoscopic third ventriculostomy in children with posterior fossa tumors: an institution experience. Turk Neurosurg 23:359-365, 2013

3. Bateman GA, Fiorentino M: Childhood hydrocephalus secondary to posterior fossa tumor is both an intra- and extraaxial process. J Neurosurg Pediatr 18:21-28, 2016

4. Bhatia R, Tahir M, Chandler CL: The management of hydrocephalus in children with posterior fossa tumours: the role of pre-resectional endoscopic third ventriculostomy. Pediatr Neurosurg 45:186-191, 2009

5. Bognár L, Borgulya G, Benke P, Madarassy G: Analysis of CSF shunting procedure requirement in children with posterior fossa tumors. Childs Nerv Syst 19:332-336, 2003

6. Cinalli G, Spennato P, Ruggiero C, Aliberti F, Zerah M, Trischitta V, et al: Intracranial pressure monitoring and lumbar puncture after endoscopic third ventriculostomy in children. Neurosurgery 58:126-136, 2006

7. Culley DJ, Berger MS, Shaw D, Geyer R: An analysis of factors determining the need for ventriculoperitoneal shunts after posterior fossa tumor surgery in children. Neurosurgery 34:402-408, 1994

8. Dewan MC, Lim J, Shannon CN, Wellons JC III: The durability of endoscopic third ventriculostomy and ventriculoperitoneal shunts in children with hydrocephalus following posterior fossa tumor resection: a systematic review and time-to-failure analysis. J Neurosurg Pediatr 19:578-584, 2017

9. Di Rocco F, Jucá CE, Zerah M, Sainte-Rose C: Endoscopic third ventriculostomy and posterior fossa tumors. World Neurosurg 79 (2 Suppl):18.e15-18.e19, 2013

10. Dias MS, Albright AL: Management of hydrocephalus complicating childhood posterior fossa tumors. Pediatr Neurosci 15:283-290, 1989
11. El Beltagy MA, Kamal HM, Taha H, Awad M, El Khateeb $\mathrm{N}$ : Endoscopic third ventriculostomy before tumor surgery in children with posterior fossa tumors, CCHE experience. Childs Nerv Syst 26:1699-1704, 2010

12. El-Gaidi MA, El-Nasr AHA, Eissa EM: Infratentorial complications following preresection CSF diversion in children with posterior fossa tumors. J Neurosurg Pediatr 15:4-11, 2015

13. El-Ghandour NMF: Endoscopic third ventriculostomy versus ventriculoperitoneal shunt in the treatment of obstructive hydrocephalus due to posterior fossa tumors in children. Childs Nerv Syst 27:117-126, 2011

14. Foreman P, McClugage S III, Naftel R, Griessenauer CJ, Ditty BJ, Agee BS, et al: Validation and modification of a predictive model of postresection hydrocephalus in pediatric patients with posterior fossa tumors. J Neurosurg Pediatr 12:220-226, 2013

15. Fritsch MJ, Doerner L, Kienke S, Mehdorn HM: Hydrocephalus in children with posterior fossa tumors: role of endoscopic third ventriculostomy. J Neurosurg 103 (1 Suppl):40-42, 2005

16. Gnanalingham KK, Lafuente J, Thompson D, Harkness W, Hayward R: The natural history of ventriculomegaly and tonsillar herniation in children with posterior fossa tumours - an MRI study. Pediatr Neurosurg 39:246-253, 2003

17. Kumar V, Phipps K, Harkness W, Hayward RD: Ventriculoperitoneal shunt requirement in children with posterior fossa tumours: an 11-year audit. Br J Neurosurg 10:467-470, 1996

18. Lin CT, Riva-Cambrin JK: Management of posterior fossa tumors and hydrocephalus in children: a review. Childs Nerv Syst 31:1781-1789, 2015

19. Morelli D, Pirotte B, Lubansu A, Detemmerman D, Aeby A, Fricx C, et al: Persistent hydrocephalus after early surgical management of posterior fossa tumors in children: is routine preoperative endoscopic third ventriculostomy justified? J Neurosurg 103 (3 Suppl):247-252, 2005

20. Riva-Cambrin J, Detsky AS, Lamberti-Pasculli M, Sargent MA, Armstrong D, Moineddin R, et al: Predicting postresection hydrocephalus in pediatric patients with posterior fossa tumors. J Neurosurg Pediatr 3:378-385, 2009

21. Romero L, Ros B, Ibáñez G, Ríus F, González L, Arráez M: Endoscopic third ventriculostomy: can we predict success during surgery? Neurosurg Rev 37:89-97, 2014

22. Ruggiero C, Cinalli G, Spennato P, Aliberti F, Cianciulli E, Trischitta V, et al: Endoscopic third ventriculostomy in the treatment of hydrocephalus in posterior fossa tumors in children. Childs Nerv Syst 20:828-833, 2004

23. Sainte-Rose C, Cinalli G, Roux FE, Maixner R, Chumas PD, Mansour M, et al: Management of hydrocephalus in pediatric patients with posterior fossa tumors: the role of endoscopic third ventriculostomy. J Neurosurg 95:791-797, 2001

24. Schijman E, Peter JC, Rekate HL, Sgouros S, Wong TT: Management of hydrocephalus in posterior fossa tumors: how, what, when? Childs Nerv Syst 20:192-194, 2004

25. Tamburrini G, Frassanito P, Bianchi F, Massimi L, Di Rocco C, Caldarelli M: Closure of endoscopic third ventriculostomy after surgery for posterior cranial fossa tumor: The "snow globe effect.” Br J Neurosurg 29:386-389, 2015

26. Tamburrini G, Massimi L, Caldarelli M, Di Rocco C: Antibiotic impregnated external ventricular drainage and third ventriculostomy in the management of hydrocephalus associated with posterior cranial fossa tumours. Acta Neurochir (Wien) 150:1049-1056, 2008

27. Tamburrini G, Pettorini BL, Massimi L, Caldarelli M, Di Rocco C: Endoscopic third ventriculostomy: the best option in the treatment of persistent hydrocephalus after posterior cranial fossa tumour removal? Childs Nerv Syst 24:14051412,2008 
28. von Elm E, Altman DG, Egger M, Pocock SJ, Gøtzsche PC, Vandenbroucke JP: The Strengthening the Reporting of Observational Studies in Epidemiology (STROBE) statement: guidelines for reporting observational studies. Lancet 370:1453-1457, 2007

29. Warf BC: Hydrocephalus in Uganda: the predominance of infectious origin and primary management with endoscopic third ventriculostomy. J Neurosurg 102 (1 Suppl):1-15, 2005

\section{Disclosures}

The authors report no conflict of interest concerning the materials or methods used in this study or the findings specified in this paper.

\section{Author Contributions}

Conception and design: Lalgudi Srinivasan. Acquisition of data: Lalgudi Srinivasan, Foster, Hennigan. Analysis and interpretation of data: Lalgudi Srinivasan, Foster. Drafting the article: Lalgudi Srinivasan. Critically revising the article: Lalgudi Srinivasan, Foster, Mallucci. Reviewed submitted version of manuscript: Lalgudi Srinivasan, van Baarsen, Pettorini, Mallucci. Approved the final version of the manuscript on behalf of all authors:

Lalgudi Srinivasan. Statistical analysis: Foster. Administrative/ technical/material support: Lalgudi Srinivasan, van Baarsen, Hennigan. Study supervision: Pettorini, Mallucci.

\section{Supplemental Information \\ Previous Presentations}

A portion of this work was presented as a platform presentation at the Society of British Neurological Surgeons 2019 spring meeting, Manchester, United Kingdom.

\section{Correspondence}

Harishchandra Lalgudi Srinivasan: Tel Aviv Sourasky Medical Center, Tel Aviv, Israel. theneurosurgeon9999@gmail.com. 